\title{
Closed versus conventional IV catheter in performing coronary CT Angiography
}

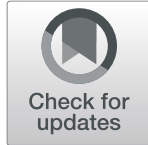

\author{
Mohamed M. Harraz ${ }^{*}$, Ahmed H. Abouissa and Wael H. Kamr
}

\begin{abstract}
Background: The purpose of this study was to compare the performance of a closed IV catheter system (CICS) with conventional IV catheters for IV contrast infusion in terms of contrast enhancement and associated complications during coronary $\mathrm{CT}$ angiography.

It is a retrospective study of 54 patients for whom a follow-up CCTA was requested were included for this study. Those patients had previous experience with the insertion of a $18 \mathrm{~g}$ conventional IV catheter which allows a flow rate of $\geq 6 \mathrm{ml} / \mathrm{s}$ and the complications during injection were registered. Patients were informed that a new IV catheter (The BD Nexiva ${ }^{\mathrm{TM}}$ Diffusic ${ }^{\mathrm{TM}}$ Closed IV Catheter System $22 \mathrm{~g}$ while still meeting a high flow rate $>6 \mathrm{~m} / \mathrm{s}$ ) will be used for their examination. We analyzed mean vascular attenuations in the ascending aorta, left main coronary artery, left ventricular (LV) cavity, and descending aorta (DA). Their feedback was collected regarding pain and the number of trials for catheter insertion. In addition, the access site was evaluated for any complication e.g. perforation, contrast extravasation or contamination.

Result: All patients were very satisfied with the new cannula. The catheter was inserted from the first trials. No one has perforation or extravasation. Mean vascular attenuations of the left main coronary artery, LV cavity, and DA were

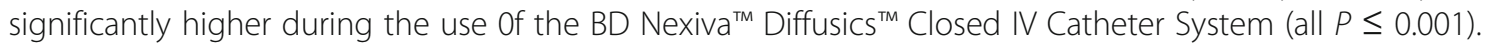

Conclusion: Although the CICS has a small lumen, it allows a high flow rate; therefore, IV access required for CCTA can be secured with higher vascular attenuation and fewer complications. It is encouraged to use the CIVC system for CT angiographic studies, in particular CCTA.
\end{abstract}

Keywords: Closed IV catheter system, Cardiac, Computed tomography angiography, MDCT

\section{Background}

The acquisition speed of current MDCT has precipitated new IV contrast infusion protocols with infusion rates higher than traditionally used, particularly for vascular imaging. IV contrast material is now routinely infused at rates between 5 and $8 \mathrm{~mL} / \mathrm{s}$ for MDCT angiography [1].

A high injection rate combined with a high iodine concentration CM is typically used in coronary CTA. An injection rate of at least $5 \mathrm{~mL} / \mathrm{s}$ is preferable, with increased rates at higher cardiac outputs [2].

Published evidence confirms that high infusion rates do not necessarily increase the rate of extravasation if the correct catheter gauge is selected. On the other

* Correspondence: harrazharraz@live.com

Radiology Department, Mansoura University Hospitals, Mansoura, Egypt hand, infusion of contrast material at a rate higher than has been specified for each catheter gauge increases the extravasation risk [3].

Infusing IV contrast material at a rate of $5 \mathrm{~mL} / \mathrm{s}$ necessitates use of at least a 20 -gauge traditional catheter to avoid exceeding the injection pressure limit and to reduce the risk of extravasation, when the infusion rate exceeds $5 \mathrm{~mL} / \mathrm{s}$, IV contrast material is ideally delivered through an 18-gauge traditional peripheral venous catheter. However, not all patients have venous access that can accommodate an 18-gauge traditional catheter [4]. Sufficient vascular enhancement is very important for CCTA to facilitate accurate detection for the coronary artery stenosis [5]. 
Achieving optimal coronary enhancement is particularly important for CCTA because coronary arteries have small caliber and tortuous course and diminution of blood flow in the presence of stenosis or obstruction. Degree of contrast enhancement relates to scan technique, patientrelated factors, and all major parameters of the injection protocol applied such as contrast media concentration, flow rate, and volume of contrast media [6].

The introduction of new technology (BD Nexiva ${ }^{\text {Tu }}$ Diffusics $^{\text {tw }}$ Closed IV Catheter System) allows the use of a smaller gauge size catheter $(22 \mathrm{~g})$ while still meeting a high flow rate $>6 \mathrm{~m} / \mathrm{s}$. This small size made puncture less painful and reduced the trials of insertion (usually single, rarely twice trials) and these were greatly appreciated by the patients with optimal vascular enhancement at the same time.

\section{Methods}

It is a retrospective study of 54 patients (30 males and 24 females ). This study was between January and December 2019. Inclusion criteria were 30 to 70-year-old patients, for whom a follow-up CCTA was requested, who had previous experience with the insertion of a 18g conventional IV catheter and had registered complications during the examination. This catheter has a diameter $=1.71 \mathrm{~mm}$ and length $=32-45 \mathrm{~mm}$ and allows a flow rate of $6 \mathrm{~mL} / \mathrm{s}$. Exclusion criteria were patients with the first-time examination, follow up patients with no previous registered complications or not registered what type of catheter were used, follow up patients with succeed insertion of conventional IV catheter from the first time in past examination, allergy to iodinated IV contrast material, pregnant, and breastfeeding women .

Patients were informed about a new IV catheter (The BD Nexiva $^{\text {tw }}$ Diffusics ${ }^{\text {Tn }}$ Closed IV Catheter System 22 g while still meeting a high flow rate $>6 \mathrm{~m} / \mathrm{s}$ ) that will be used for the study. As a regular prerequisite, consent was obtained for contrast administration. Other parameters e.g. the access vein (right antecubital), CM concentration (omnipaque 350 $\mathrm{mg} / \mathrm{mL})$, volume $(70-80 \mathrm{mls})$, and flow rate $(6 \mathrm{~mL} / \mathrm{s})$ and the automatic injector (Medrad Stellent CT Injection System) were the same.

The new fenestrated IV catheter system used the 22gauge BD Nexiva Diffusics (Fig. 1) was recently available (Becton Dickinson Infusion Systems Inc, UT, USA). In contrast to a non-fenestrated catheter, the diffusics has multiple diffuser holes in the wall of the catheter, just proximal to the tip. It has $0.9 \mathrm{~mm}$ diameter and $25 \mathrm{~mm}$ length and allows a high flow rate up to $6 \mathrm{~mL} / \mathrm{s}$ with automatic injectors.

\section{CT technique}

All patients underwent CCTA with a 128 section CT scanner (Siemens Somatom Definition AS) MDCT

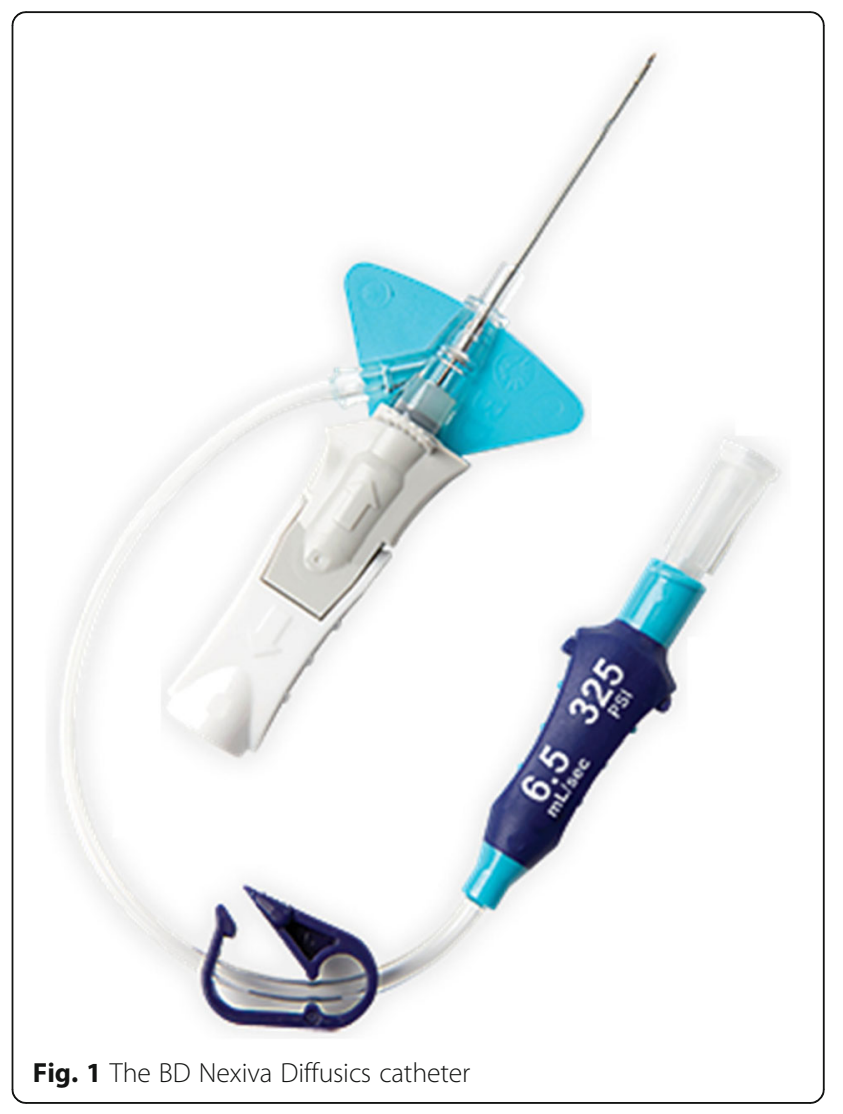

scanner. Before CCTA, all patients with a baseline heart rate greater than 70 beats/min received IV esmolol 10 to $30 \mathrm{mg}$. For the patients without contraindications to nitroglycerin, nitroglycerin $0.6 \mathrm{mg}$ was immediately administered sublingually before contrast injection. CCTA was applied with 270-ms rotation time, 100-kV-tube voltage, and an automatic tube current modulation. During CCTA acquisition, a bolus of omnipaque $350 \mathrm{mg} /$ $\mathrm{mL}$, volume (70-80 mls) was injected intravenously (6 $\mathrm{mL} / \mathrm{s}$ ) followed by a $50-\mathrm{mL}$ saline chaser. A region of interest was placed in the ascending thoracic aorta, and image acquisition was automatically initiated once a selected threshold had been reached with bolus tracking. All scans were performed with prospective electrocardiogram gating. Vessel attenuations (in HUs) were measured by one reader, who was blinded to the types of catheter, using circular regions of interest manually placed in the following regions: ascending aorta, left main coronary artery, left ventricular (LV) cavity, and descending aorta at the level of the left bronchus.

\section{Statistical analysis}

At the end of the CCTA, the patient's feedback was collected regarding pain and the number of trials during catheter insertion. In addition, the access site was evaluated for any complication, e.g., perforation or contamination during 
insertion or contrast extravasation during the injection. We analyzed mean vascular attenuations in the ascending aorta, left main coronary artery, left ventricular (LV) cavity, and descending aorta (DA).The Kruskal-Wallis test and Student's $t$ test for continuous variables were used to compare characteristics and contrast enchantment in each examination. All statistical tests were performed using the SPSS statistical software package.

\section{Results}

All patients were happy and very satisfied with the new cannula (Table 1). The catheter was inserted from the first or maximum of two trials. No one has perforation or extravasation. No blood leakage during insertion, therefore, reducing exposure to blood and infection. Three patients were physicians, they appreciated the cannula and advocated for regular use. Our experience with the new BD Nexiva ${ }^{\text {Tw }}$ integrated IV catheter system is limited to diagnostic radiological investigation which requires high injection rate, in particular CCTA. The BD Nexiva $^{\text {Tix }}$ Diffusics ${ }^{\text {Ti }}$ Closed IV Catheter System allows the use of a smaller gauge size catheter $(22 \mathrm{~g})$ while still meeting a high flow rate $>6 \mathrm{~m} / \mathrm{s}$. It allowed for contrast administration at the required protocol rates with a smaller gauge size and it was effective in all patients. The CIVC reduced the calls for catheter insertion assistance. It also reduced the patients' waiting time for catheter placement. In the evaluation of the ability of a 22gauge BD Nexiva ${ }^{\text {Tu }}$ Diffusics ${ }^{\text {Tn }}$ Closed IV Catheter System, mean vascular attenuations of the left main coronary artery, LV cavity, and descending aorta were significantly higher in the 22-gauge BD Nexiva ${ }^{\text {Tu }}$ Diffusics ${ }^{\text {Ta }}$ Closed IV Catheter System than in the 18-gauge conventional IV catheter $(P=0.015,0.028,0.001$, respectively); however, mean vascular attenuations of the ascending aorta were not different $(P=0.665)$ (Fig. 2).

Table 1 Comparison of complications between 18-gauge nonfenestrated IV catheter and 22-gauge fenestrated IV catheter

\begin{tabular}{lll}
\hline Finding & $\begin{array}{l}\text { 18-gauge traditional } \\
\text { peripheral venous } \\
\text { catheter }\end{array}$ & $\begin{array}{l}\text { 22-gauge (BD Nexiva } \\
\text { Diffusics } \\
\text { Catheter System) }\end{array}$ \\
\hline $\begin{array}{l}\text { Pain during } \\
\text { catheter insertion. }\end{array}$ & 54 & 3 \\
$\begin{array}{l}\text { Number of trials } \\
\text { for catheter } \\
\text { insertion }\end{array}$ & 29 after 3rd trial & 51 at first trial \\
$\begin{array}{l}\text { Access site } \\
\text { perforation }\end{array}$ & 23 & 3 after 2nd trial \\
$\begin{array}{l}\text { Access site } \\
\text { contrast } \\
\text { extravasation }\end{array}$ & 23 & 0 \\
$\begin{array}{l}\text { Access site } \\
\text { contamination }\end{array}$ & 10 & 0 \\
\hline
\end{tabular}

\section{Discussion}

CT angiography quality is affected by many factors related to temporal, spatial, and contrast resolution. Contrast resolution is affected by the quality of contrast enhancement, which relates to the iodine delivery rate and scan timing. Iodine delivery rate can be increased by using higher concentration contrast agents or higher infusion rates. Multiple studies have shown that the contrast infusion rate directly affects the arterial enhancement level and image quality $[7,8]$. Not all patients can tolerate highcontrast infusion rates, however, owing to the quality of their venous access. Accordingly, IV catheter selection and the IV contrast infusion rate are tailored to the individual patient's vein quality $[9,10]$.

In our study, we are focus on proper IV catheter selection during CCTA as in our experience it is important for patients and image quality with few types of research in this subject. One of the basic components of good venous access is the IV catheter. CCTA requires good peripheral venous access which in turn depends on a good-sized vein and large bore IV catheter to allow a high flow of contrast and the saline chaser. Regularly, a $18 \mathrm{~g}$ IV catheter is used to allow infusion of contrast at $\geq 5 \mathrm{~mL} / \mathrm{s}$. A right antecubital vein is preferred access to avoid streak artifacts that may affect image quality if a left vein is used $[3,11,12]$.

A new peripheral IV catheter ( BD-Nexiva closed IV catheter) is intended for use with a power injection system. One important goal of the design was to create a small (22 G and $24 \mathrm{G}$ ) peripheral IV catheter device capable of safely delivering power injection flow rates. The catheter system has increased pressure capability in addition to diffuser holes in the catheter tip which improves catheter stability and decreases tip jet velocity compared to a standard IV catheter. Furthermore, the diffuser holes reduce system pressure at the tip of the catheter during open-flow conditions [13, 14].

In our study we found that successful catheter placement on the first attempt was 51/54 patients at the first trial and 3/54 after 2nd trial for 22-gauge fenestrated catheter however no cases from the first trial, 25/54 after 2nd trial and 29/54 after 3rd trial for 18-gauge non-fenestrated catheter. Also, no cases of contrast extravasation with 22-gauge fenestrated catheter however 23 cases with an 18-gauge nonfenestrated catheter and these agree with Johnson et al. [9] that also provides evidence that the presence of fenestrations reduces the risk of venous rupture and extravasation by directing flow from a single end hole to across multiple side holes and the end hole, thus enabling a higher contrast infusion rate.

In our study, mean vascular attenuations of the left main coronary artery, LV cavity, and descending aorta were higher in the 22-gauge fenestrated than in the 18- 


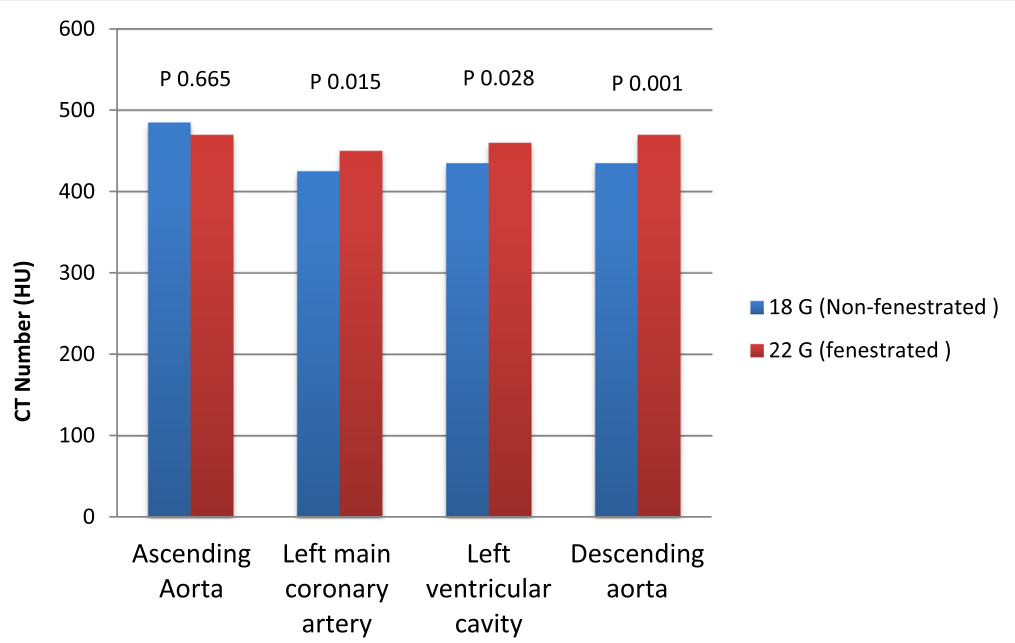

Fig. 2 Comparison of mean vascular attenuation between 18-gauge non-fenestrated IV catheter and 22-gauge fenestrated IV catheter

gauge non-fenestrated catheter (Fig. 3). In our study, mean vascular attenuations of the ascending aorta were not different. This finding can be explained by the use of bolus tracking for cardiac MDCT and fenestrated catheter. We used ascending aorta for bolus tracking method and short scan delay. Therefore, mean vascular attenuation of the ascending aorta was not statistically different despite the shorter time to peak enhancement in the fenestrated catheter and all this agrees with Kim et al. [15].

A number of studies have shown that the contrast infusion rate directly affects the arterial enhancement level and image quality for vascular imaging [16-18]. Therefore, we speculate that a fenestrated catheter will be a useful method for cardiac MDCT especially in patients with poor quality of venous access.
Our study has a limitation that it was a retrospective study with a small sample size; however, our patient group size is still considerable because there are few investigations comparing fenestrated and non-fenestrated peripheral venous catheters for CCTA.

In this study, we report our experience of the closed IV catheter during CCTA regarding, mean vascular attenuation as well as patient's feedback. The small diameter 22gauge fenestrated catheter which allows an injection rate of $>6 \mathrm{~m} / \mathrm{s}$ is the most cheerful advantage perceived by the patients. Mean vascular attenuations were higher and in this small size made puncture less painful, reduced the trials of insertion (usually single, rarely twice trials), easier, faster insertion, and almost complication-free, e.g., perforation, contrast extravasation or contamination and this agree with Tamura et al. [19].

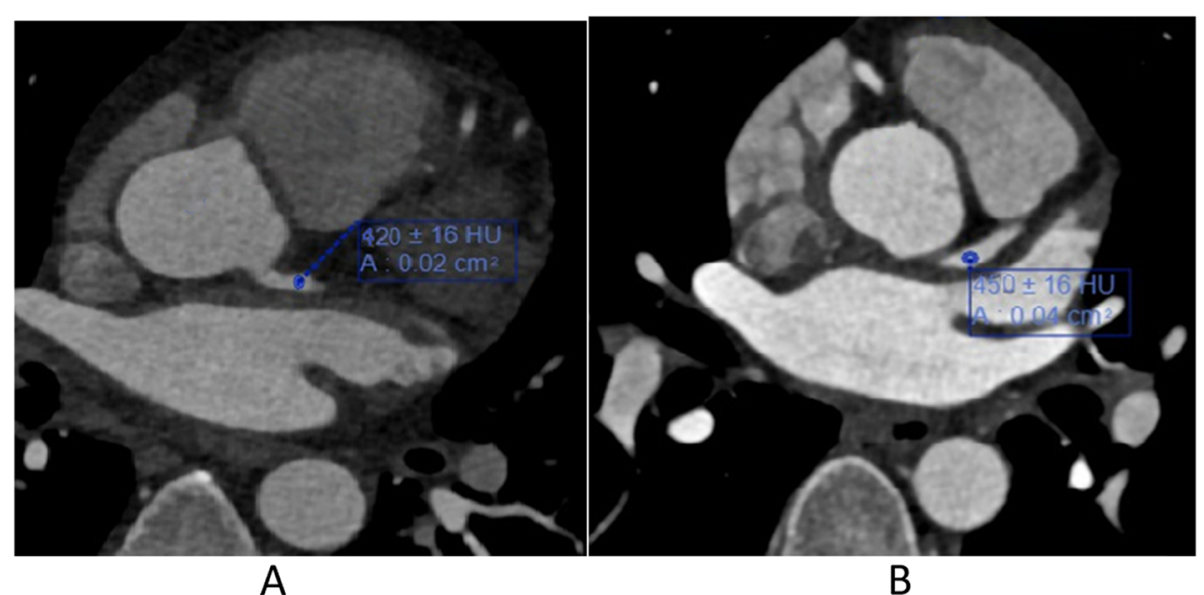

Fig. 3 a CCTA scan of a 44-year-old male, with atypical chest pain using conventional IV catheter 18 G. Axial image showing vessel attenuation at the region of interest (ROI) in the lumen of the left main coronary artery about $420 \mathrm{HU}$. $\mathbf{b}$ Comparative image of the same patient with follows up CCTA scan using the new closed IV Catheter $22 \mathrm{G}$ with the same protocol. Axial image showing vessel attenuation at the ROI in the lumen of the same left main coronary artery was higher about $450 \mathrm{HU}$ 


\section{Conclusion}

The new catheter technology demonstrated the ability to utilize a smaller gauge catheter that successfully meets flow rate and image quality requirements. Additionally, improvements were noted in number of insertion attempts, patient's wait time with virtually complicationfree access during coronary CTA.

\section{Abbreviations \\ MDCT: Multi-detector computed tomography; CICS: Closed IV catheter system; CCTA: Coronary CT angiography; CM: Contrast media; LV: Left ventricle; DA: Descending aorta; IV: Intravenous; ROI: Region of interest}

\section{Acknowledgements}

Not Applicable

\section{Authors' contributions}

MMH carried out the radiological studies and participated in design of the study. AHA collected the patients' data and performed the statistical analysis. WHK participated in the sequence alignment and drafted the manuscript. $\mathrm{MMH}$ participated in the acquisition of data. AHA participated in the sequence alignment. WHK participated in the design of the study and performed the statistical analysis. MMH conceived of the study, and participated in its design and coordination and helped to draft the manuscript. AHA wrote the paper with revision. All authors read and approved the final manuscript.

\section{Funding}

This study had no funding from any resource.

\section{Availability of data and materials}

The datasets used and/or analyzed during the current study are available from the corresponding author on reasonable request.

\section{Ethics approval and consent to participate}

This study was approved by the Research Ethics Committee of Alnoor Hospital in Saudi Arabia. All patients included in this study gave written informed consent to participate in this research. The committee's reference number is not available.

\section{Consent for publication}

All patients included in this research gave written informed consent to publish the data contained within this study.

\section{Competing interests}

The authors declare that they have no competing interests

Received: 17 March 2020 Accepted: 27 May 2020

Published online: 09 June 2020

\section{References}

1. Silvennoinen HM, Hamberg LM, Lindsberg PJ et al (2008) CT perfusion identifies increased salvage of tissue in patients receiving intravenous recombinant tissue plasminogen activator within 3 hours of stroke onset. AJNR 29:1118-1123

2. Abbara S, Arbab-Zadeh A, Callister TQ et al (2009) SCCT guidelines for performance of coronary computed tomographic angiography: A report of the Society of Cardiovascular Computed Tomography Guidelines Committee. J Cardiovasc Comput Tomogr 3:190-204

3. Wienbeck S, Fischbach R, Kloska SP et al (2010) Prospective study of access site complications of automated contrast injection with peripheral venous access in MDCT. AJR 195:825-829

4. Johnson PT, Christensen GM, Fishman EK et al (2014) IV contrast administration with dual source 128-MDCT: a randomized controlled study comparing 18-gauge nonfenestrated and 20-gauge fenestrated catheters for catheter placement success, infusion rate, image quality, and complications. Am J Roentgenol 202:1166-1170

5. Pugliese $F(2008)$ Which contrast agent for coronary $C T$ angiography? RAD Magazine 34:33-34. 38
6. Bae KT (2010) Intravenous contrast medium administration and scan timing at CT: considerations and approaches. Radiology 256:32-61

7. Behrendt FF, Bruners P, Keil S et al (2010) Effect of different saline chaser volumes and flow rates on intravascular contrast enhancement in CT using a circulation phantom. Eur J Radiol 73:688-693

8. Schueller G, Schima W, Schueller-Weidekamm C et al (2006) Multidetector CT of pancreas: effects of contrast material flow rate and individualized scan delay on enhancement of pancreas and tumor contrast. Radiology 241:441-448

9. Johnson PT, Christensen G, Lai H et al (2014) Catheter insertion for intravenous (IV) contrast infusion in multidetector row computed tomography (MDCT): Defining how catheter caliber selection impacts procedure of catheter insertion, IV contrast infusion rate, complication rate, and MDCT image quality. J Comput Assist Tomogr 38:281-284

10. Davenport MS, Wang CL, Bashir MR et al (2012) Rate of contrast material extravasations and allergic-like reactions: effect of extrinsic warming of lowosmolality iodinated CT contrast material to 37 degrees C. Radiology 262: 475-484

11. Schwab SA, Uder M, Anders K et al (2009) Peripheral intravenous power injection of iodinated contrast media through $22 \mathrm{G}$ and $20 \mathrm{G}$ cannulas: can high flow rates be achieved safely? A clinical feasibility study. Rofo 181:355-361

12. Tomizawa N, Suzuki F, Akahane M et al (2013) Effect of saline flush on enhancement of proximal and distal segments using 320-row coronary CT angiography. Eur J Radiol 82:1255-1259

13. Marin D, Nelson RC, Rubin GD et al (2011) Body CT: technical advances for improving safety. AJR 197:33-41

14. Grigioni M, Daniele C, Morbiducci U et al (2002) Computational model of the fluid dynamics of a cannula inserted in a vessel: incidence of the presence of side holes in blood flow. J Biomech 35:1599-1612

15. Kim J, Kim EJ, Hur JH et al (2019) The Usefulness of Fenestrated Intravenous Catheters Compared With Nonfenestrated Catheter for Cardiac Multidetector Computed Tomography. J Comput Assist Tomogr 43(3):423-427

16. Mihl C, Kok M, Wildberger JE et al (2015) Coronary CT angiography using low concentrated contrast media injected with high flow rates: feasible in clinical practice. Eur J Radiol 84:2155-2160

17. Wang CL, Cohan RH, Ellis JH et al (2007) Frequency, management, and outcome of extravasation of nonionic iodinated contrast medium in 69,657 intravenous injections. Radiology 243:80-87

18. Cademartiri F, Mollet NR, Lemos PA et al (2006) Higher intracoronary attenuation improves diagnostic accuracy in MDCT coronary angiography. AJR Am J Roentgenol 187:430-433

19. Tamura A, Kato K, Kamata M et al (2017) Selection of peripheral intravenous catheters with 24-gauge side-holes versus those with 22-gaugeend-hole for MDCT: a prospective randomized study. Eur J Radiol 87:8-12

\section{Publisher's Note}

Springer Nature remains neutral with regard to jurisdictional claims in published maps and institutional affiliations.

\section{Submit your manuscript to a SpringerOpen ${ }^{\circ}$ journal and benefit from:}

- Convenient online submission

- Rigorous peer review

- Open access: articles freely available online

- High visibility within the field

- Retaining the copyright to your article

Submit your next manuscript at $>$ springeropen.com 